\title{
Anomalous $\mathcal{U}(1)$ : Solving Various Puzzles

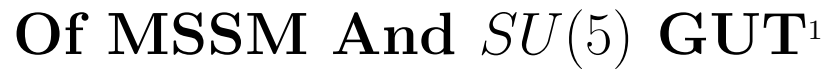

\author{
Qaisar Shafi ${ }^{a}$ f, Zurab Tavartkiladze ${ }^{b, c}$ 色 \\ ${ }^{a}$ Bartol Research Institute, University of Delaware, Newark, DE 19716, USA \\ ${ }^{b}$ Institute for Theoretical Physics, Heidelberg University, Philosophenweg 16, \\ D-69120 Heidelberg, Germany \\ c Institute of Physics, Georgian Academy of Sciences, Tbilisi 38007r, Georgia
}

\begin{abstract}
We discuss how an anomalous $\mathcal{U}(1)$ symmetry when appended to MSSM and SUSY GUTs [e.g. $S U(5)]$ can help overcome a variety of puzzles related to charged fermion masses and mixings, flavor changing processes, proton decay and neutrino oscillations. Proton lifetime for $S U(5) \mathrm{GUT}$, in particular, is predicted in a range accessible to the ongoing or planned searches.
\end{abstract}

\footnotetext{
${ }^{1}$ To appear in the proceedings of NOON2001 Workshop held in Kashiwa, Japan, 5-8 Dec. 2001.

${ }^{2}$ E-mail address: shafi@bartol.udel.edu

${ }^{3}$ E-mail address: Z.Tavartkiladze@ThPhys.Uni-Heidelberg.DE
} 


\section{Introduction: Some Puzzles of MSSM and Beyond}

The standard model provides an excellent description of almost all present experimental data. Supersymmetry (SUSY) is highly motivated because it offers possibility of resolving the gauge hierarchy problem. Furthermore, superstring theories are believed to be good candidates for a unified description of gauge theories and quantum gravity. Therefore, for realistic model building including SUSY is a good way to proceed and SUSY GUTs provide an excellent example. However, supersymmetry introduces several problems and puzzles which require explanations. The puzzles can be divided in two categories: 1 puzzles which are common only to SUSY theories, i.e. those which appear due to SUSY extension (SUSY puzzles); and $\mathbf{2}$ The puzzles which exist also in non-SUSY theories and within SUSY extensions have the same status (non-SUSY puzzles). From puzzles 1, 2 we list here only those which we will attempt to resolve.

\section{SUSY puzzles}

a) Problem of FCNC arises because with SUSY there is a new source for flavor violation. Apriori, without any specific arrangement there is no universality and an alignment (which would guarantee flavor conservation) in the soft SUSY breaking terms. This can create non-diagonal gaugino-fermion-sfermion interactions, leading to the FCNC [1].

b) $d=5$ Baryon number violation;

With SUSY there is a new source for baryon number violation. Namely $d=5$ operators [2]

$$
\frac{\lambda}{M} q q q l, \quad \frac{\lambda^{\prime}}{M} u^{c} u^{c} d^{c} e^{c}
$$

where $M$ is some cutoff mass scale and $\lambda, \lambda^{\prime}$ are dimensionless couplings depending on the model. In GUTs, $M$ usually stands for colored triplet masses. In minimal $S U(5)$ the nucleon decay mainly proceeds through the channel $p \rightarrow K \nu$ and its lifetime is estimated to be $10^{29 \pm 2}$ yr [3], [4], which is embarrassingly small in comparison with the latest SuperKamiokande (SK) limit $\tau_{p}^{\exp } \gtrsim 10^{33} \mathrm{yr}$ [5]. Therefore, some mechanism (with reasonable extension) [4], [6], [7] must be applied to suppress these contributions. Once colored triplet induced $d=5$ operators are properly suppressed, one has to take care of Planck scale $d=5$ baryon number violating operators. In (1) with $M=M_{P l}=$ $2.4 \cdot 10^{18} \mathrm{GeV}$, in order to satisfy the experimental bounds we need $\lambda, \lambda^{\prime} \lesssim 10^{-8}$. This kind of suppression requires additional explanation [4], [8]-[13].

\section{Non-SUSY puzzles}

a) Problem of flavor - hierarchies between charged fermion masses and mixings;

In the charged fermion sector there are noticeable hierarchies within the fermion Yukawa couplings and the CKM matrix elements. Since the mass of the top quark is close to the electroweak symmetry breaking scale $(\sim 100 \mathrm{GeV})$, its Yukawa coupling is of order unity $\left(\lambda_{t} \sim 1\right)$. As far as the Yukawa couplings of the $b$ quark and $\tau$ lepton 
are concerned, their values could vary in a range $\lambda_{b} \sim \lambda_{\tau} \sim 10^{-2}-1$, depending on the value of the MSSM parameter $\tan \beta(\sim 1-60)$. Introducing the dimensionless parameter $\epsilon \simeq 0.2$, one can express the observed hierarchies between the charged fermion Yukawa couplings as follows:

$$
\begin{gathered}
\lambda_{t} \sim 1, \quad \lambda_{u}: \lambda_{c}: \lambda_{t} \sim \epsilon^{6}: \epsilon^{3}: 1 \\
\lambda_{b} \sim \lambda_{\tau} \sim \lambda_{t} \frac{m_{b}}{m_{t}} \tan \beta, \quad \lambda_{d}: \lambda_{s}: \lambda_{b} \sim \epsilon^{5}: \epsilon^{2}: 1 \\
\lambda_{e}: \lambda_{\mu}: \lambda_{\tau} \sim \epsilon^{5}: \epsilon^{2}: 1
\end{gathered}
$$

while for the CKM matrix elements:

$$
V_{u s} \sim \epsilon, \quad V_{c b} \sim \epsilon^{2}, \quad V_{u b} \sim \epsilon^{3} .
$$

In constructing models, one should arrange for a natural understanding of the hierarchies in (2) $-($ (5) .

b) Atmospheric and Solar Neutrino puzzles;

The latest atmospheric and solar neutrino data (see [14 and 15 respectively) seem to provide convincing evidence for the phenomena of neutrino oscillations. Ignoring the LSND data, the atmospheric and solar neutrino anomalies can be explained within the three states of active neutrinos. In this paper we will study oscillation scenarios without the sterile neutrinos, which are disfavored by the data [15, 14.

The atmospheric neutrino data suggest oscillations of $\nu_{\mu}$ into $\nu_{\tau}$, with the following oscillation parameters:

$$
\begin{gathered}
\mathcal{A}\left(\nu_{\mu} \rightarrow \nu_{\tau}\right) \equiv \sin ^{2} 2 \theta_{\mu \tau} \simeq 1 \\
\Delta m_{\mathrm{atm}}^{2} \simeq 3 \cdot 10^{-3} \mathrm{eV}^{2} .
\end{gathered}
$$

The solar neutrino anomaly seems consistent with oscillation scenarios, amongst which the most likely seems to be the large angle MSW (LMA) oscillation of $\nu_{e}$ into $\nu_{\mu, \tau}$ [15], with the oscillation parameters:

$$
\begin{gathered}
\mathcal{A}\left(\nu_{e} \rightarrow \nu_{\mu, \tau}\right) \equiv \sin ^{2} 2 \theta_{e \mu, \tau} \approx 0.8, \\
\Delta m_{\mathrm{sol}}^{2} \sim 10^{-4} \mathrm{eV}^{2} .
\end{gathered}
$$

The scenario of low MSW (LOW) oscillations of solar neutrinos require:

$$
\begin{gathered}
\sin ^{2} 2 \theta_{e \mu, \tau} \simeq 1.0, \\
\Delta m_{\mathrm{sol}}^{2} \simeq 8 \cdot 10^{-8} \mathrm{eV}^{2} .
\end{gathered}
$$

Let us note that the small angle MSW and large angle vacuum oscillation solutions seem to be disfavored by data. 
It is worth noting that within MSSM, the neutrinos acquire masses only through nonrenormalizable $d=5$ Planck scale operators $l_{i} l_{j} h_{u}^{2} / M_{P}$ which, for $\left\langle h_{u}^{0}\right\rangle \sim 100 \mathrm{GeV}$ and $M_{P}=2.4 \cdot 10^{18} \mathrm{GeV}$ (reduced Planck mass) give $m_{\nu_{i}} \sim 10^{-5} \mathrm{eV}$. Therefore, for (6) or (17) we need physics beyond the MSSM. In order to generate the appropriate neutrino masses, we will introduce heavy right handed neutrino states $\mathcal{N}_{i}$. The 'light' left-handed neutrinos will acquire masses through the see-saw mechanism [16].

In building neutrino oscillation scenarios, the main challenge is to generate desirable magnitudes for neutrino masses and their mixings, and to understand why in some cases the mixing angles are large (and even maximal), while the quark CKM matrix elements (5) are suppressed. Below we will present a mechanism which successfully resolves all of these problems.

c) Wrong asymptotic relations originating from GUTs.

Within GUTs the quark-lepton families are embedded in unified multiplets and because of this, wrong asymptotic relations for masses and mixings are possible unless some care is exercised. In minimal $S U(5)$ chiral matter consists of anomaly free $10+\overline{5}$ supermultiplets per generation, where $10=\left(q, u^{c}, e^{c}\right), \overline{5}=\left(l, d^{c}\right)$. The couplings generating the up, down quark and charged lepton masses are respectively $10 \cdot 10 \cdot H+10 \cdot \overline{5} \cdot \bar{H},(H, \bar{H}$ are 'higgs' superfields in 5 and $\overline{5}$ representations). The second coupling gives $\hat{M}_{f}^{0}=\hat{M}_{d}^{0}$ at GUT scale, which for the third generation yields the reasonable asymptotic relation

$m_{b}^{0}=m_{\tau}^{0}$, but for light generations it gives $\left(\frac{m_{d}}{m_{s}}\right)^{0}=\left(\frac{m_{e}}{m_{\mu}}\right)^{0}$ which is unacceptable. For improving this picture, either a scalar 45 plet [17], or vector-like matter [18], [7], or some non-renormalizable operators [19] can be employed.

As we see, for solving the problems listed above, an extension of the minimal scheme is needed. Otherwise, in some cases, we should simply assume the presence of appropriately suppressed couplings and mass scales [for instance for 1.b), 2.a),b)]. The latter puzzle is tied to the so-called naturalness issue [20], namely why are some couplings and scales small, when apriori there is no reason to expect it? Below we discuss some extensions which provide natural mechanisms for resolving the above-listed problems.

\section{Anomalous $\mathcal{U}(1)$ as a Flavor Symmetry and Medi- ator of SUSY Breaking}

We introduce a $\mathcal{U}(1)$ gauge symmetry which acts as a flavor symmetry and provides for a natural understanding of the hierarchies between charged fermion masses and mixings. $\mathcal{U}(1)$ also turns out to be crucial for building textures of neutrino mass matrices that provide scenarios for simultaneous explanations of atmospheric and solar neutrino puzzles. It will turn out that $\mathcal{U}(1)$ is anomalous, which allows us to use it as a mediator of SUSY breaking [21], [22]. Thanks to this, the squarks and sleptons which correspond to the 
light generations can have masses of few $\cdot 10 \mathrm{TeV}$ (this value can be acceptable also for $\tilde{b}^{c}, \tilde{\nu}_{\tau}$ and $\tilde{\tau}$ in the low $\tan \beta$ regime). Because of this, the FCNC can be adequately suppressed due to the decoupling [23]- 26], [22]. It turns out that together with this, some nucleon decay modes are strongly suppressed [26], [22]. Namely, diagrams with heavy squarks and/or sleptons inside the loops decouple. As far as the Planck scale $d=5$ baryon number violating operators are concerned, they can also be adequately suppressed by the $\mathcal{U}(1)$ symmetry. Therefore, the advantages of $\mathcal{U}(1)$ symmetry are manyfold, and we will present an $S U(5)$ GUT to show how things work out.

The anomalous $\mathcal{U}(1)$ factors can arise from string theories 1 . Cancellation of the anomaly occurs through the Green-Schwarz mechanism [27]. Due to the anomaly the Fayet-Illiopoulos D-term $\xi \int d^{4} \theta V_{A}$ is always generated, where in string theory $\xi$ is given by 28

$$
\xi=\frac{g_{A}^{2} M_{P}^{2}}{192 \pi^{2}} \operatorname{Tr} Q
$$

The $D_{A}$-term will have the form:

$$
\frac{g_{A}^{2}}{8} D_{A}^{2}=\frac{g_{A}^{2}}{8}\left(\Sigma Q_{a}\left|\varphi_{a}\right|^{2}+\xi\right)^{2}
$$

where $Q_{a}$ is the 'anomalous' charge of $\varphi_{a}$ superfield. For $\mathcal{U}(1)$ breaking we introduce the singlet superfield $X$ with $\mathcal{U}(1)$ charge $Q_{X}$. Assuming $\xi>0[\operatorname{Tr} Q>0$ in (9)], and taking

$$
Q_{X}=-1
$$

the cancellation of $D_{A}$ in (10) and nonzero $\langle X\rangle$ are ensured $(\langle X\rangle=\sqrt{\xi})$. Further, we will take

$$
\frac{\langle X\rangle}{M_{P}} \equiv \epsilon \simeq 0.2
$$

where $\epsilon$ turns out to be an important expansion parameter. Let us note that an anomalous $\mathcal{U}(1)$ for understanding the hierarchies of fermion masses and mixings and a variety of neutrino oscillation scenarios has been discussed in several papers of [29]-35], [7], [22], [18, 36, [11]

\subsection{Neutrino oscillations and quark-lepton masses}

Let us begin with the neutrino sector and we first discuss two ways of obtaining large/maximal neutrino mixings with the help of $\mathcal{U}(1)$ flavor symmetry. With two flavors of lepton doublets $l_{1}$ and $l_{2}$, one way of having large mixing is the so-called democratic approach. Here

\footnotetext{
${ }^{4}$ Recently, ref. 13 presented an example where anomalous $\mathcal{U}(1)$ arises in $4 \mathrm{D}$ level through $5 \mathrm{D}$ orbifold compactification. The cancellation of anomalies occur through bulk Chern-Simons term.
} 
the $\mathcal{U}(1)$ symmetry does not distinguish the two flavors [29], i.e. they have the same $\mathcal{U}(1)$ charges $Q_{l_{1}}=Q_{l_{2}}=n$ (positive integer number). In this case, the expected neutrino mass matrix will be:

$$
\hat{m}_{\nu}=\left(\begin{array}{ll}
1 & 1 \\
1 & 1
\end{array}\right) \bar{m} \epsilon^{2 n}, \quad \bar{m}=\frac{h_{u}^{2}}{M}
$$

with entries of order unity $\left(\bar{M}\right.$ is some mass scale and we have assumed $\left.Q_{h_{u}}=0\right)$. Therefore, naturally large $\nu_{1}-\nu_{2}$ mixing is expected, $\sin ^{2} 2 \theta_{12} \sim 1$. Also, one can expect $m_{\nu_{1}} \sim m_{\nu_{2}}$, and if this mechanism is used for atmospheric neutrinos, somehow one has to keep one state light, in order to accomodate also the solar neutrino puzzle. This can be done [30, 18] by introducing a single right handed neutrino $\mathcal{N}$. After integrating it out, due to degeneracy only one state acquires a non-zero mass. The remaining states can be used for the solar neutrino puzzle. An appropriate mass scale for the latter can be generated by introducing a relatively heavy right handed state $\mathcal{N}^{\prime}$ with suppressed coupling to $\mathcal{N}$.

A different approach is the so-called maximal mixing mechanism [31], [36], [1]]. It is realized by assigning different $\mathcal{U}(1)$ charges for the flavors $l_{1}, l_{2}$. Introducing two right handed states $\mathcal{N}_{1}, \mathcal{N}_{2}$ and the following $\mathcal{U}(1)$ charge prescriptions

$$
\begin{gathered}
Q_{l_{1}}=k+n, \quad Q_{l_{2}}=k, \quad Q_{h_{u}}=0 \\
Q_{\mathcal{N}_{1}}=-Q_{\mathcal{N}_{2}}=k+k^{\prime}
\end{gathered}
$$

with $k, n, k^{\prime}>0, n \geq k^{\prime}$, the 'Dirac' and 'Majorana' coupling are given by:

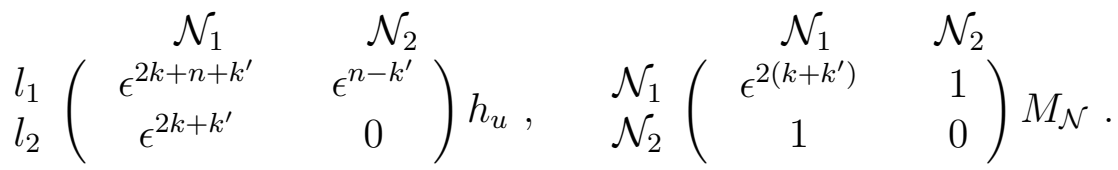

After integrating out the heavy $\mathcal{N}_{1}, \mathcal{N}_{2}$ states, the neutrino mass matrix is given by

$$
\hat{m}_{\nu}=\left(\begin{array}{cc}
\epsilon^{n} & 1 \\
1 & 0
\end{array}\right) \bar{m}, \quad \bar{m}=\frac{h_{u}^{2} \epsilon^{2 k+n}}{M_{\mathcal{N}}}
$$

a quasi off-diagonal form, leading to a mixing angle

$$
\sin ^{2} 2 \theta_{12}=1-\mathcal{O}\left(\epsilon^{2 n}\right)
$$

which is close to maximal mixing. The form (16) is guaranteed by the appropriate zero entries in (15), which are ensured by $\mathcal{U}(1)$ symmetry. This mechanism turns out to be 
very convenient for achieving nearly maximal mixings between neutrino flavors within various realistic models, such as $S U(5)$ [18], $S O(10)$ [35], $S U(4)_{c} \times S U(2)_{L} \times S U(2)_{R}$ [1], etc.

Returning to our scheme, we attempt to obtain the bi-maximal texture through $\mathcal{U}(1)$ flavor symmetry. For this, we will combine the two mechanisms discussed above. Namely, the second and third lepton doublet states will have the same $\mathcal{U}(1)$ charges, which will lead to their large mixing. The state $l_{1}$ will have a suitable charge, one that ensures maximal $\nu_{1}-\nu_{2}$ mixing.

Introducing two right handed $\mathcal{N}_{1,2}$ neutrino states and choosing $\mathcal{U}(1)$ charges as

$$
\begin{gathered}
Q_{X}=-1, Q_{l_{2}}=Q_{l_{3}}=k, Q_{l_{1}}=k+n, Q_{h_{u}}=Q_{h_{d}}=0, \\
Q_{\mathcal{N}_{1}}=-Q_{\mathcal{N}_{2}}=k+k^{\prime},
\end{gathered}
$$

with

$$
k, n, k^{\prime}>0, \quad n \geq k^{\prime},
$$

the 'Dirac' and 'Majorana' couplings will have forms:

$$
\begin{array}{cc}
\mathcal{N}_{1} & \mathcal{N}_{2} \\
l_{1} \\
l_{2} \\
l_{3}
\end{array}\left(\begin{array}{cc}
\epsilon^{2 k+n+k^{\prime}} & \epsilon^{n-k^{\prime}} \\
\epsilon^{2 k+k^{\prime}} & 0 \\
\epsilon^{2 k+k^{\prime}} & 0
\end{array}\right) h_{u}, \quad \mathcal{N}_{1}\left(\begin{array}{cc}
\mathcal{N}_{1} & \mathcal{N}_{2} \\
\epsilon^{2\left(k+k^{\prime}\right)} & 1 \\
1 & 0
\end{array}\right) M_{\mathcal{N}}
$$

After integrating out $\mathcal{N}_{1,2}$, we obtain the texture

$$
\hat{M}_{\nu} \propto\left(\begin{array}{ccc}
\epsilon^{n} & 1 & 1 \\
1 & 0 & 0 \\
1 & 0 & 0
\end{array}\right) m, \quad m=\frac{\epsilon^{2 k+n} h_{u}^{2}}{M_{\mathcal{N}}},
$$

In (21) coefficients of order unity are assumed. Without $(1,1)$ entry the (21) has $L_{e}-L_{\mu}-$ $L_{\tau}$ symmetry, which also can be used [37] for obtaining bi-maximal texture. In our case deviation from $(1,1)$ zero entry is controlled by $\mathcal{U}(1)$ flavor symmetry [34]. The nonzero $(1,1)$ entry in (21) guarantees that $\Delta m_{12}^{2} \neq 0$. Using (21) the oscillation parameters are:

$$
\begin{gathered}
\Delta m_{32}^{2} \equiv m_{\mathrm{atm}}^{2}=m^{2} \sim 10^{-3} \mathrm{eV}^{2}, \\
\mathcal{A}\left(\nu_{\mu} \rightarrow \nu_{\tau}\right) \sim 1, \\
\Delta m_{21}^{2} \simeq 2 m_{\mathrm{atm}}^{2} \epsilon^{n} \\
\mathcal{A}\left(\nu_{e} \rightarrow \nu_{\mu, \tau}\right)=1-\mathcal{O}\left(\epsilon^{2 n}\right) .
\end{gathered}
$$


Note that the model does not constrain $n$ for the time being. So, LMA and LOW solutions for solar neutrinos can be realized. With prescription (18), the expected contribution from the charged lepton sector to the angles $\theta_{23}^{l}$ and $\theta_{12}^{l}$ will be $\sim 1$ and $\sim \epsilon^{n}$ respectively. These do not alter the expressions in (22), (23).

The $\mathcal{U}(1)$ charge selection in (18) nicely blends with the charged fermion sector. Indeed, considering the following prescription:

$$
\begin{gathered}
Q_{q_{3}}=0, \quad Q_{q_{2}}=2, \quad Q_{q_{1}}=3, \quad Q_{d_{3}^{c}}=Q_{d_{2}^{c}}=p+k, \\
Q_{d_{1}^{c}}=p+k+2, \quad Q_{u_{3}^{c}}=0, \quad Q_{u_{2}^{c}}=1, \quad Q_{u_{1}^{c}}=3, \\
Q_{e_{3}^{c}}=p, \quad Q_{e_{2}^{c}}=p+2, \quad Q_{e_{1}^{c}}=p+5-n,
\end{gathered}
$$

the structures of Yukawa matrices for up-down quarks and charged leptons are respectively:

$$
\begin{gathered}
q_{1}\left(\begin{array}{rrr}
u_{1}^{c} & u_{2}^{c} & u_{3}^{c} \\
\epsilon^{6} & \epsilon^{4} & \epsilon^{3} \\
\epsilon^{5} & \epsilon^{3} & \epsilon^{2} \\
\epsilon^{3} & \epsilon & 1
\end{array}\right) h_{u}, \\
q_{3} \\
q_{1}\left(\begin{array}{ccc}
d_{1}^{c} & d_{2}^{c} & d_{3}^{c} \\
\epsilon^{5} & \epsilon^{3} & \epsilon^{3} \\
\epsilon^{4} & \epsilon^{2} & \epsilon^{2} \\
\epsilon^{2} & 1 & 1
\end{array}\right) \epsilon^{p+k} h_{d}, \\
q_{1}\left(\begin{array}{ccc}
e_{1}^{c} & e_{2}^{c} & e_{3}^{c} \\
l_{2} & \epsilon^{n+2} & \epsilon^{n} \\
l_{3} & \epsilon^{5-n} & 1 \\
\epsilon^{5-n} & \epsilon^{2} & 1
\end{array}\right) \epsilon^{p+k} h_{d} .
\end{gathered}
$$

Upon diagonalization of (25)-(27) it is easy to verify that the desired relations (2)-(5) for the Yukawa couplings and CKM matrix elements are realized. From (26), (27) we have

$$
\tan \beta \sim \epsilon^{p+k} \frac{m_{t}}{m_{b}}
$$

As we previously mentioned, MSSM does not fix the values of $n, k, p$ in (18), (24). Because of this, the solar neutrino oscillation scenario is not specified and both LMA and LOW are possible solutions. 


\section{2 $\quad D_{A}$-term SUSY Breaking Suppression of FCNC and Nucleon Decay}

The cancellation of $D_{A}$-term (10) was ensured by the VEV of $X$ (at this stage we do not consider any superpotential couplings for $X$ ). Including a mass term for $X$ in the superpotential,

$$
W_{m}=\frac{m}{2} X^{2}
$$

the cancellation of $D_{A}$ will be partial, and SUSY will be broken due to non-zero $F$ and $D$ terms. Taking into account (10) and (29), we have

$$
X^{2}=\xi-\frac{4 m^{2}}{g_{A}^{2}}, \quad\left\langle D_{A}\right\rangle=\frac{4 m^{2}}{g_{A}^{2}}, \quad\left\langle F_{X}\right\rangle \simeq m \sqrt{\xi} .
$$

From (10), taking into account (30), for the soft scalar masses squared $\left(\operatorname{mass}^{2}\right)$ we have

$$
m_{\tilde{\varphi}_{a}}^{2}=Q_{a} m^{2}
$$

Thus, the scalar components of superfields which have non-zero $\mathcal{U}(1)$ charges gain masses through $\left\langle D_{A}\right\rangle$. We will assume that the scale $m$ is in the range $\sim 10 \mathrm{TeV}$. Those states which have zero $\mathcal{U}(1)$ charges will gain soft masses of the order of gravitino mass $m_{3 / 2}$ from the Kähler potential

$$
m_{3 / 2}=\frac{F_{X}}{\sqrt{3} M_{P}}=m \frac{\epsilon}{\sqrt{3}},
$$

which, for $m=10 \mathrm{TeV}$, is relatively suppressed $(\sim 1 \mathrm{TeV})$. The gaugino masses also will have the same magnitudes

$$
M_{\tilde{G}_{i}} \sim m_{3 / 2} \sim 1 \mathrm{TeV} .
$$

The mass term (29) violates the $\mathcal{U}(1)$ symmetry and is taken to be in the $10 \mathrm{TeV}$ range. Its origin may lie in a strong dynamics where $m$ is replaced by the VEV of some superfield(s). We do not present here any examples of this and refer the reader to [21], 222 for detailed discussions. The important point is that the soft masses (31) of sparticles are controlled by the $\mathcal{U}(1)$ symmetry.

Turning now to the question of FCNC, we require that the 'light' quark-lepton superfields carry non-zero $\mathcal{U}(1)$ charges. This means that the soft masses of their scalar components are in the $10 \mathrm{TeV}$ range, which automatically suppresses flavor changing processes such as $K^{0}-\bar{K}^{0}, \mu \rightarrow e \gamma$ etc., thereby satisfying the present experimental bounds [1]. To prevent upsetting the gauge hierarchy, the third generation up squarks must have masses no larger than a $\mathrm{TeV}$ or so [24] (hence they have zero $\mathcal{U}(1)$ charge). The same applies to sbottom and stau for large $\tan \beta$ since, for $\lambda_{b} \sim \lambda_{\tau} \sim 1$, large masses $(\gtrsim 10 \mathrm{TeV})$ of $\tilde{b}$ and $\tilde{\tau}$ would spoil the gauge hierarchy. 
Although the tree level mass of the stop can be arranged to be in the $1 \mathrm{TeV}$ range by the $\mathcal{U}(1)$ symmetry, the 2-loop contributions from heavy sparticles of the first two generations can drive the stop mass $^{2}$ negative [24]. This is clearly unacceptable, and one proposal for avoiding it 25] requires the existence of new states in the multi-TeV range. This type of extension makes the decoupled solution viable and realistic models can be built [22].

Let us now turn to some implications for proton decay. We assume that $d=5$ baryon number violating operators arise from the couplings

$$
q A q T+q B l \bar{T}
$$

after integration of color triplets $T, \bar{T}$ with mass $M_{T} \sim 2 \cdot 10^{16} \mathrm{GeV}$ (we first consider triplet couplings with left-handed matter). After wino dressing of appropriate $d=5$ operators, the resulting $d=6$ operators causing proton to decay into a neutrino and charged lepton channels have the respective forms [3], [4]:

$$
\begin{aligned}
& \frac{g_{2}^{2}}{M_{T}} \alpha\left(u_{a} d_{b}^{i}\right)\left(d_{c}^{j} \nu^{k}\right) \varepsilon^{a b c}, \\
& \frac{g_{2}^{2}}{M_{T}} \alpha^{\prime}\left(u_{a} d_{b}^{i}\right)\left(u_{c} e^{j}\right) \varepsilon^{a b c},
\end{aligned}
$$

where

$$
\begin{gathered}
\alpha=-\left[\left(L_{d}^{+} \hat{B} L_{e}\right)_{j k}\left(L_{u}^{+} \hat{A} L_{d}^{*}\right)_{m n}+\left(L_{d}^{+} \hat{A} L_{u}^{*}\right)_{j m}\left(L_{d}^{+} \hat{B} L_{e}\right)_{n k}\right] V_{m i}\left(V^{+}\right)_{n 1} I\left(\tilde{u}^{m}, \tilde{d}^{n}\right)+ \\
{\left[\left(L_{u}^{+} \hat{A} L_{d}^{*}\right)_{1 i}\left(L_{u}^{+} \hat{B} L_{e}\right)_{m k}-\left(L_{d}^{+} \hat{A} L_{u}^{*}\right)_{i m}\left(L_{u}^{+} \hat{B} L_{e}\right)_{i k}\right] V_{m j} I\left(\tilde{u^{m}}, \tilde{e^{k}}\right)} \\
\alpha^{\prime}=\left[-\left(L_{u}^{+} \hat{A} L_{d}^{*}\right)_{1 i}\left(L_{d}^{+} \hat{B} L_{e}\right)_{m j}+\left(L_{u}^{+} \hat{A} L_{d}^{*}\right)_{1 m}\left(L_{d}^{+} \hat{B} L_{e}\right)_{i j}\right]\left(V^{+}\right)_{m 1} I\left(\tilde{d^{m}}, \tilde{\nu^{j}}\right)+ \\
{\left[\left(L_{u}^{+} \hat{B} L_{e}\right)_{1 j}\left(L_{u}^{+} \hat{A} L_{d}^{*}\right)_{m n}+\left(L_{u}^{+} \hat{A} L_{d}^{*}\right)_{1 m}\left(L_{e}^{T} \hat{B}^{T} L_{u}^{*}\right)_{j n}\right]\left(V^{+}\right)_{m 1} V_{n i} I\left(\tilde{d}^{m}, \tilde{u}^{n}\right) .}
\end{gathered}
$$

$L$ 's are unitary matrices which rotate the left handed fermion states to diagonalize the mass matrices, and I's are functions obtained after loop integration and depend on the SUSY particle masses circulating inside the loop. For example [3],

$$
I(\tilde{u}, \tilde{d})=\frac{1}{16 \pi^{2}} \frac{m_{\tilde{W}}}{m_{\tilde{u}}^{2}-m_{\tilde{d}}^{2}}\left(\frac{m_{\tilde{u}}^{2}}{m_{\tilde{u}}^{2}-m_{\tilde{W}}^{2}} \ln \frac{m_{\tilde{u}}^{2}}{m_{\tilde{W}}^{2}}-\frac{m_{\tilde{d}}^{2}}{m_{\tilde{d}}^{2}-m_{\tilde{W}}^{2}} \ln \frac{m_{\tilde{d}}^{2}}{m_{\tilde{W}}^{2}}\right)
$$

with similar expressions for $I(\tilde{d}, \tilde{\nu})$ and $I(\tilde{u}, \tilde{e})$.

Consider those diagrams in which sparticles of the first two families participate. Since their masses are large $(\gtrsim 10 \mathrm{TeV})$ compared to the case with minimal $N=1$ SUGRA, 
we expect considerable suppression of proton decay. For minimal $N=1$ SUGRA, $m_{\tilde{u}} \sim$ $m_{\tilde{d}} \sim m_{\tilde{W}} \sim m_{3 / 2} \sim 1 \mathrm{TeV}$, and (39) can be approximated by

$$
I_{0} \approx \frac{1}{16 \pi^{2}} \frac{1}{m_{3 / 2}}
$$

In the $\mathcal{U}(1)$ mediated SUSY breaking scenario, expression (39) takes the form

$$
I^{\prime} \approx \frac{1}{16 \pi^{2}} \frac{m_{\tilde{W}}}{m_{\tilde{q}}^{2}} \equiv \eta I_{0}
$$

The nucleon lifetime in this case will be enhanced by the factor $\frac{1}{\eta^{2}} \sim 10^{4}$.

Of course, there exist diagrams in which one sparticle from the third and one from the 'light' families participate. In this case, (39) takes the form

$$
I^{\prime \prime} \approx \frac{1}{16 \pi^{2}} \frac{2 m_{\tilde{W}}}{m_{\tilde{q}}^{2}} \ln \frac{m_{\tilde{q}}}{m_{\tilde{W}}} \equiv \eta^{\prime} I_{0}
$$

and the corresponding proton lifetime will be $\sim \frac{1}{\eta^{22}} \sim 500$ times larger. This suppression is enough to bring the proton lifetime near the experimental limit, providing an opportunity for testing this type of scenario in the near future.

As pointed out in [3], [4] (within minimal $N=1$ SUGRA), the contribution from diagrams in which sparticles from the third generation participate could be comparable with those arising from the light generation sparticle exchange. Namely, second term of (37) with $\tilde{t}, \tilde{\tau}$ inside the loop give a contribution comparable to diagrams with first two generation sparticles circulating inside the loop. Since minimal SUSY $S U(5)$ gives unacceptably fast proton decay with $\tau_{0} \sim 10^{29 \pm 2} \mathrm{yr}$, care must be exercised in realistic model building (the situation is exacerbated if $\tan \beta$ is large). This problem is easily avoided in the anomalous $\mathcal{U}(1)$ mediated SUSY breaking scenario. Note that in second term of (37) the $I$ depends on mass of $\tilde{e}^{k}$ state and even if the latter belongs to the third family, it can have mass in the $10 \mathrm{TeV}$ range if $\tan \beta$ is either of intermediate $(\sim 10-15)$ or low value (this is required for preserving the desired gauge hierarchy). The contributions coming from first term of (37) could be adequately suppressed due to CKM matrix elements [note that first term in (37) contains extra multiplier $\left(V^{+}\right)_{n 1}$ ]. In sect. 3, for $S U(5)$ GUT example we will precisely show this. Due to same reasons the contributions from terms of (38) are suppressed. Since these terms would induce $p \rightarrow K \mu$ decays, the additional inhensiment factor (of the order of 10) in proton lifetime come from the hadronic matrix element, which correspond to proton decay with emission of charged lepton.

As far as the right handed $d=5$ operators $u^{c i} u^{c j} d^{c m} e^{c n}$ are concerned, the dominant contribution from them comes through higgsino dressings. Due to antisymmetry in respect 
of color in this $d=5$ operator, $u^{c}$ states should be taken from the different generations. So, either $c^{c}$ or $t^{c}$ will appear. Thay must not be appeared in an external line after dressing, otherwise the diagram would not be relevant for nucleon decay. Since first two generation sparticles and also all $\tilde{d}^{c}{ }^{i}$ states are in $10 \mathrm{TeV}$ range, relevant diagram would be that one which inside the loop contain $\tilde{t}^{c}$ and $\tilde{\tau}^{c}$ states (the latter is neccessarily light within $S U(5)$ GUT, because it comes from $10_{3}$ plet). Due to suppressed mixings of third generation states with first and second generations and also due to small Yuakawas (in small $\tan \beta$ regime) appearing due to higgsino dressing, the suppression of nucleon decay can be guaranteed also for this case [this is shown in sect. 3 on an $S U(5)$ example].

In general, within this scenario universality does not hold and nucleon decays through gluino dressings would occur. However, heavy sparticles will still play a crucial role in suppression of nucleon decay. Which contribution is dominant depends on the details of the scenario and in sect. 3 we study this issue within a realistic $S U(5)$ model.

Thus, thanks to the anomalous $\mathcal{U}(1)$ symmetry, in addition to avoiding dangerous FCNC, one can also obtain adequate suppression of nucleon decay. Interestingly, this disfavors the large $\tan \beta$ regime which could be a characteristic feature of this class of models.

\subsection{Possible neutrino oscillation scenarios}

As we previously mentioned, MSSM does not fix the values of $n, k, p$ in (18), (24). Because of this, the solar neutrino oscillation scenario is not specified. According to (23) both LMA and LOW are possible solutions. Namely, for $n=3$ we have $\Delta m_{12}^{2} \sim 10^{-5} \mathrm{eV}^{2}$, which corresponds to LMA. $n=6$ gives $\Delta m_{12}^{2} \sim 10^{-7} \mathrm{eV}^{2}$, which is the scale for the LOW solution

In SUSY SU(5) GUT, due to unified 10, $\overline{5}$ multiplets:

$$
Q_{q}=Q_{e^{c}}=Q_{u^{c}}=Q_{10}, \quad Q_{l}=Q_{d^{c}}=Q_{\overline{5}}
$$

Hierarchies of the CKM matrix elements in (5) dictate the relative $\mathcal{U}(1)$ charges of the 10-plets

$$
Q_{10_{3}}=0, \quad Q_{10_{2}}=2, \quad Q_{10_{1}}=3,
$$

while the Yukawa hierarchies (2)-(四), together with (44), require that

$$
Q_{\overline{5}_{3}}=Q_{\overline{5}_{2}}=k, \quad Q_{\overline{5}_{1}}=k+2 .
$$

Comparing (43)-(45) with (18), (24) we see that the minimal $S U(5)$ GUT fixes $n$ and $p$ as

$$
n=2, \quad p=0 \text {. }
$$


The mass squared splitting in (23) then equals $\Delta m_{12}^{2} \sim 10^{-4} \mathrm{eV}^{2}$, which is a reasonable scale for LMA scenario. Therefore, realisation of our bi-maximal mixing scenario in the framework of $S U(5)$ GUT dictates that the LMA scenario is responsible for the solar neutrino deficit (more detailed discussion of $S U(5)$ GUT will be presented in the next section). The same conclusion can be reached for $S O(10)$ GUT where we have three 16-plets of chiral supermultiplets which unify the quark-lepton superfields. We do not present the details here but refer the reader to [35], where an explicit $S O(10)$ model with anomalous $\mathcal{U}(1)$ flavor symmetry is considered for explanations of fermion masses, their mixings, as well as neutrino anomalies.

For models in which an anomalous flavor $\mathcal{U}(1)$ also provides SUSY breaking soft masses, from (31) we should require $Q_{\tilde{q}, \tilde{l}}>0$. On the other hand, from (23), the realization of LMA and LOW respectively require $n=3$ and $n=6$ (if $p \geq 1$ ), both of which guarantee $Q_{e^{c}} \geq 0$. Therefore, a scenario in which an anomalous flavor $\mathcal{U}(1)$ mediates SUSY breaking permits LMA and LOW oscillations for solar neutrinos (the large angle vacuum oscillation would not be realized within this scenario, since it requires $n=10$ giving negative $Q_{e^{c}}$.

\section{An $S U(5)$ Example}

Let us now consider in detail a SUSY SU(5) GUT and show how things discussed in the previous section work out in practice. We also present here the possibility for avoiding the problematic asymptotic mass relations $\hat{M}_{d}^{0}=\hat{M}_{e}^{0}$ (for first and second families).

The three families of matter in $(10+\overline{5})$ representations have the transformation properties as given in (44), (45), while the scalar superfields $\bar{H}(\overline{5})+H(5)$ have $Q_{\bar{H}}=Q_{H}=0$. The couplings relevant for the generation of up, down quark and charged lepton masses respectively are given by

$$
\begin{aligned}
& \begin{array}{l}
10_{1} \\
10_{2} \\
10_{3}
\end{array}\left(\begin{array}{ccc}
10_{1} & 10_{2} & 10_{3} \\
\epsilon^{6} & \epsilon^{5} & \epsilon^{3} \\
\epsilon^{3} & \epsilon^{4} & \epsilon^{2} \\
\epsilon^{2} & 1
\end{array}\right) H \\
& \begin{array}{l}
\overline{5}_{1} \\
10_{1} \\
10_{2} \\
10_{3}
\end{array}\left(\begin{array}{ccc}
\epsilon^{5} & \overline{5}_{3} & \epsilon^{3} \\
\epsilon^{4} & \epsilon^{2} & \epsilon^{2} \\
\epsilon^{2} & 1 & 1
\end{array}\right) \epsilon^{k} \bar{H} .
\end{aligned}
$$

Upon diagonalization of (47), (48) we obtain the desirable hierarchies (2)-(5) and $\lambda_{b} \sim$ $\lambda_{\tau} \sim \epsilon^{k}$. 
The reader will note, however, that (48) implies the asymptotic mass relations $\hat{M}_{d}^{0}=$ $\hat{M}_{e}^{0}$, which are unacceptable for the two light families. This is readily avoided through the mechanism suggested in [18], [7] by employing two pairs of $(\overline{15}+15)_{1,2}$ matter states. Namely, with $\mathcal{U}(1)$ charges

$$
Q_{15_{1}}=-Q_{\overline{15_{1}}}=3, \quad Q_{15_{2}}=-Q_{\overline{15_{2}}}=2,
$$

consider the couplings

$$
\overline{15}_{1}\left(\begin{array}{ccc}
10_{1} & 10_{2} & 10_{3} \\
1 & 0 & 0 \\
\epsilon & 1 & 0
\end{array}\right) \Sigma, \quad \overline{15}_{1}\left(\begin{array}{cc}
15_{1} & 15_{2} \\
1 & 0 \\
\epsilon & 1
\end{array}\right) M_{15},
$$

where $\Sigma$ is the scalar 24-plet whose VEV breaks $S U(5)$ down to $S U(3)_{c} \times S U(2)_{L} \times U(1)_{Y}$. For $M_{15} \sim\langle\Sigma\rangle$, we see that the 'light' $q_{1,2}$ states reside both in $10_{1,2}$ and $15_{1,2}$ states with similar 'weights'. At the same time, the other light states from 10-plets $\left(u^{c}\right.$ and $\left.e^{c}\right)$ will not be affected because the 15-plets do not contain fragments with the relevant quantum numbers. Thus, the relations $m_{s}^{0}=m_{\mu}^{0}$ and $m_{d}^{0}=m_{e}^{0}$ are avoided, while $m_{b}^{0}=m_{\tau}^{0}$ still holds since the terms in (50) do not affect $10_{3}$.

As far as the sparticle spectrum is concerned, since the superfields $10_{3}, \bar{H}, H$ have zero $\mathcal{U}(1)$ charges, the soft masses of their scalar components will be in the $1 \mathrm{TeV}$ range,

$$
m_{\tilde{10}_{3}} \sim m_{\bar{H}} \sim m_{H} \sim m_{3 / 2}=1 \mathrm{TeV},
$$

while for $10_{1,2}$ and $\overline{5}_{1}$ we have

$$
m_{\tilde{10}_{1}} \sim m_{\tilde{10}_{2}} \sim m_{\tilde{\overline{5}}_{1}} \sim m \sim 10 \mathrm{TeV} .
$$

The soft masses of the scalar fragments from $\overline{5}_{2,3}$ depend on the value of $k$, and for $k \neq 0$, they also will be in the $10 \mathrm{TeV}$ range, which is preferred for proton stability. As far as neutrino oscillations are concerned, as already pointed out in sect. 1.3, due to $S U(5)$ and $\mathcal{U}(1)$ charge prescriptions, the LMA solution is preferred with the texture in (21).

\subsection{Nucleon Decay in $S U(5)$}

Turning to the issue of nucleon decay in $S U(5)$, we will take $k \neq 0$ in (45), which provides soft masses for $\overline{5}_{2,3}$ states in the $10 \mathrm{TeV}$ range. Let us first make sure that this ensures proton stability. As pointed out in sect. 2, diagrams with slepton inside the loop $\left[2^{\text {nd }}\right.$ term of (37) and $1^{\text {st }}$ term of (38)] are appropriately suppressed.

The diagrams corresponding to the first terms in (37) induce nucleon decay with the dominant mode $p \rightarrow K \nu_{\mu, \tau}$ with $\tilde{t}$ and $\tilde{b}$ running in the loop. For our scenario, taking 
into account the couplings (47), (48), (50), the dominant contribution accompanying $I$ (from first term in (37)) is of order order of $\epsilon^{k+2} \lambda_{t} V_{t d} V_{u b} \sim \lambda_{s} \lambda_{t} V_{t d} V_{u b}$. The corresponding partial lifetime in units of $\tau_{0} \equiv \tau[$ min. SUSY $S U(5)]$ will be $\tau\left(p \rightarrow K \nu_{\mu, \tau}\right)=\tau_{0}\left(\frac{\lambda_{c} \sin ^{2} \theta_{c}}{\lambda_{t} V_{t d} V_{u b}}\right)^{2}$. For the CKM matrix elements we have

$$
V_{t d}=0.004-0.014, \quad V_{u b}=0.0025-0.0048,
$$

and for their central values we have $\tau\left(p \rightarrow K \nu_{\mu, \tau}\right)=217 \tau_{0}$, which presumably is still compatible with the available limits (of course, for lower values in (53) we can have much longer lived nucleon $\left.\tau\left(p \rightarrow K \nu_{\mu, \tau}\right) \sim 2 \cdot 10^{3} \tau_{0}\right)$.

The second term in (38) induces the decay $p \rightarrow K \mu$. It can be verified that the suppression discussed above occurs in this case too. Furthermore, additional inhancement of the partial lifetime by a factor of order 10 occurs from the hadronic matrix element corresponding to proton decay with emission of charged lepton.

As far as the right handed $u^{c} u^{c} d^{c} e^{c}$ type $d=5$ operators are concerned, due to arguments presented at the end of sect. 2, the relevant $d=5$ operator will be $\tilde{\tau}^{c} \tilde{t}^{c} u^{c} s^{c}$ which in our $S U$ (5) model, according to (47), (48), (50) will appear as $\frac{1}{M_{T}} \lambda_{t} \lambda_{b} \epsilon^{3} \cdot \tilde{\tau}^{c} \tilde{t}^{c} u^{c} s^{c}$. After higgsino dressing relevant $d=6$ operator will have the form

$$
\frac{\kappa}{M_{T}} \lambda_{t}^{2} \lambda_{b}^{2} \epsilon^{3} V_{t d} \cdot\left(\overline{u^{c}} \overline{s^{c}}\right)\left(d \nu_{\mu, \tau}\right) \text {. }
$$

$\kappa$ is factor which coincides with those appearing in minimal SUSY SU(5) (we assume that $M_{\tilde{W}} \simeq \mu$-term). Operator (54) leads to $p \rightarrow K \nu_{\mu, \tau}$ and corresponding partial lifetime is $\tau\left(p \rightarrow \nu_{\mu, \tau}\right)=\left(\frac{g_{2}^{2} \lambda_{s} \lambda_{c} \sin ^{2} \theta_{c}}{\lambda_{b}^{2} \lambda_{t}^{2} \epsilon^{3} V_{t d}}\right)^{2} \tau_{0}$, which for $\lambda_{b} \sim 10^{-2}(\tan \beta \sim 1)$ and $V_{t d}=0.004$ gives $\tau\left(p \rightarrow K \nu_{\mu, \tau}\right) \sim 400 \tau_{0}$, compatible with experimental data.

In considered $S U(5)$ GUT due to different $\mathcal{U}(1)$ charges the universality of soft mass ${ }^{2}$ is violated. Because of this nucleon decay through gluino dressings will not be canceled (as is the case in minimal $N=1$ SUGRA). So, these diagrams are important since the proton decay amplitude will be increased by the factor $\alpha_{3} / \alpha_{2}$. For our $S U(5)$ scenario the dominant contributions come from diagrams inside which run the third generation sparticles (namely $\tilde{t}, \tilde{b}$ from $q_{3}$ ). The relevant $d=5$ operators, obtained after integration of colored triplet higgs fields, will be

$$
\begin{gathered}
\frac{1}{M_{T}} \lambda_{t} \lambda_{b} \epsilon^{3} \cdot \tilde{t} \tilde{b} d \nu_{\mu, \tau}, \quad \frac{1}{M_{T}} \lambda_{t} \lambda_{b} \epsilon^{2} \cdot \tilde{t} \tilde{s} d \nu_{\mu, \tau}, \\
\frac{1}{M_{T}} \lambda_{t} \lambda_{b} \epsilon \cdot \tilde{t} \tilde{b} u \mu,
\end{gathered}
$$

which, after gluino dressings, lead to the following four-fermion operators:

$$
\frac{\kappa}{M_{T}} \alpha_{3} \lambda_{t} \lambda_{b} \epsilon^{2} L_{13}^{u}\left[\epsilon L_{23}^{d} \cdot\left(d \nu_{\mu, \tau}\right)(u s)+L_{13}^{d} \cdot\left(s \nu_{\mu, \tau}\right)(u d)\right],
$$




$$
\frac{\kappa}{M_{T}} \alpha_{3} \lambda_{t} \lambda_{\mu} \epsilon L_{13}^{u} L_{23}^{d} \cdot(u \mu)(u s)
$$

$L^{u, d}$ are unitary matrices that rotate the left-handed up and down quark flavor states. Operators (57) and (58) respectively lead to the decays $p \rightarrow K \nu_{\mu, \tau}, p \rightarrow K \mu$, and the corresponding lifetimes are

$$
\begin{aligned}
& \tau\left(p \rightarrow K \nu_{\mu, \tau}\right)=\left[\frac{\alpha_{2}}{\alpha_{3}} \frac{\lambda_{s} \lambda_{c} \sin ^{2} \theta_{c}}{\lambda_{b} \lambda_{t} \epsilon^{3} L_{13}^{u} L_{23}^{d}}\right]^{2} \tau_{0}, \\
& \tau(p \rightarrow K \mu)=10\left[\frac{\alpha_{2}}{\alpha_{3}} \frac{\lambda_{s} \lambda_{c} \sin ^{2} \theta_{c}}{\lambda_{\mu} \lambda_{t} \epsilon L_{13}^{u} L_{23}^{d}}\right]^{2} \tau_{0} .
\end{aligned}
$$

For $V_{c b}$ we have

$$
V_{c b}=0.036-0.046
$$

which together with (53) can dictate that the values of $L^{u, d}$ can vary,

$$
L_{12}^{u}, L_{13}^{d}=(1.8-3.5) \cdot 10^{-3}, \quad L_{23}^{d}=(2-4) \cdot 10^{-2} .
$$

Taking the lowest values from (62), the nucleon lifetimes are

$$
\tau\left(p \rightarrow K \nu_{\mu, \tau}\right) \sim 177 \tau_{0}, \quad \tau(p \rightarrow K \mu) \sim 2 \cdot 10^{3} \tau_{0}
$$

which are still compatible with the experimental bounds f, with the dominant decay mode being $p \rightarrow K \nu_{\mu, \tau}$.

Before concluding, let us note that the Planck scale suppressed baryon number violating $d=5$ operator $\frac{1}{M_{P}} q_{1} q_{1} q_{2} l_{2,3}$, which could cause unacceptably fast proton decay, is also suppressed, since it emerges from the coupling

$$
\frac{1}{M_{P}}\left(\frac{X}{M_{P}}\right)^{8+k} 10_{1} 10_{1} 10_{2} \overline{5}_{2,3},
$$

with the suppression guaranteed by the $\mathcal{U}(1)$ symmetry.

\section{Conclusions}

It is quite remarkable that the introduction of an anomalous $\mathcal{U}(1)$ symmetry within a supersymmetric setting can have several far reaching consequences. In particular, the flavor and dimension five proton decay problems encountered in SUSY models can be

\footnotetext{
${ }^{5}$ The lifetimes can be further increased if say $L_{23}^{d}$ is more suppressed and in the appropriate entry of CKM matrix the main contribution comes from $L^{u}$.
} 
overcome. The atmospheric and solar neutrino puzzles can be nicely explained via neutrino oscillations. Although we have emphasized the $S U(5)$ model here, the discussion can be extended to other realistic models such as those based on $S O(10)$.

\section{Acknowledgements}

We are very grateful to the organizers of the NOON2001 workshop, especially Professor M. Bando, for creating a very stimulating environment at the conference and for their generous hospitality. We also thank the organizers of the post-NOON workshop in Kyoto.

\section{References}

[1] F. Gabbiani, E. Gabrielli, A. Masiero, L. Silvestrini, Nucl.Phys. B477 (1996) 321; G. Eyal, A. Masiero, Y. Nir, L. Silvestrini, hep-ph/9908382.

[2] S. Weinberg, Phys. Rev. D 26 (1982) 287; N. Sakai, T. Yanagida, Nucl. Phys. B 197 (1982) 533.

[3] P. Nath, R. Arnowitt, Phys. Rev. D 38 (1988) 1479; J. Hisano, H. Murayama, T. Yanagida, Nucl. Phys. B 402 (1993) 46.

[4] V. Ben-Hamo, Y. Nir, Phys. Lett. B 339 (1994) 77; L. Hall, H. Murayama, Phys. Rev. Lett. 75 (1995) 3985; Z. Berezhiani, hep-ph/9602325; C. Carone, et al., Phys. Rev. D 54 (1996) 2328; Z. Berezhiani, Z. Tavartkiladze, M. Vysotsky, hep-ph/9809301.

[5] Particle Data Group, Phys. Rev. D 66 (2002) 010001.

[6] G. Dvali, Phys. Lett. B 287 (1992) 101; K. Babu, S. Barr, Phys. Rev. D 48 (1993) 5354; J. Hisano, et al., Phys. Lett. B 342 (1995) 138; J. Pati, Phys. Lett. B 288 (1996) 532; I. Gogoladze, A. Kobakhidze, hep-ph/9610389; Y. Achiman, C. Merten, hep-ph/9911314; Z. Chacko, R. Mohapatra, Phys. Rev. D 59 (1999) 011702; Q. Shafi, Z. Tavartkiladze, Nucl. Phys. B 573 (2000) 40.

[7] Q. Shafi, Z. Tavartkiladze, Phys. Lett. B 459 (1999) 563.

[8] L. Ibanez, G. Ross, Nucl. Phys. B 368 (1992) 3.

[9] I. Antoniadis, hep-th/0102202.

[10] I. Antoniadis, et al., Phys. Lett. B 194 (1987) 231; G. Lazarides, C. Panagiotakopoulos, Q. Shafi, Phys. Lett. B 315 (1993) 325; G. Dvali, Q. Shafi, Phys. Lett. B 403 (1997) 65; Q. Shafi, Z. Tavartkiladze, Nucl. Phys. B 552 (1999) 67. 
[11] Q. Shafi, Z. Tavartkiladze, Nucl. Phys. B 549 (1999) 3.

[12] M. Kakizaki, M. Yamaguchi, hep-ph/0203192.

[13] C.A. Lee, Q. Shafi, Z. Tavartkiladze, hep-ph/0206258.

[14] S. Fukuda et al. [Super-Kamiokande Collaboration], Phys. Rev. Lett. 88 (2000) 3999; N. Fornengo et al., Nucl. Phys. B 580 (2000) 58.

[15] S. Fukuda et al. [Super-Kamiokande Collaboration], Phys. Lett. B 539 (2002) 179; J. Bahcall, P. Krastev, A. Smirnov, hep-ph/0006078; M.C. Gonzalez-Garcia et al., Nucl. Phys. B 573 (2000) 3.

[16] M. Gell-Mann, P. Ramond and R. Slansky, in: Supergravity, eds. P. van Nieuwenhuizen and D. Freedman (North-Holland, Amsterdam, 1979) p. 315; T. Yanagida, Prog. Th. Phys. B 135 (1979) 66; R. Mohapatra and G. Senjanovic, Phys. Rev. Lett. 44 (1980) 912.

[17] H. Gorgi, C. Jarlskog, Phys. Lett. B 88 (1979) 279; J. Harvey, P. Ramond, D. Reiss, Phys. Lett. B 92 (1980) 309; S. Dimopoulos, L.J. Hall, S. Raby, Phys. Rev. Lett. 68 (1992) 1984, Phys. Rev. D 45 (1992) 4192; V. Barger et. al., Phys. Rev. Lett. 68 (1992) 3394; H. Arason et. al., Phys. Rev. D 47 (1993) 232.

[18] Q. Shafi, Z. Tavartkiladze, Phys. Lett. B 451 (1999) 129.

[19] J. Ellis, M. Gaillard, Phys. Lett. B 88 (1979) 315; R. Barbieri, D. Nanopoulos, Phys. Lett. B 95 (1980) 43; Z. Berezhiano, hep-ph/9602325; G. Altarelli, F. Feruglio, hepph/9812475; see also references therein; Q. Shafi, Z. Tavartkiladze, Phys. Lett. B 522 (2001) 102 .

[20] S. Weinberg, Phys. Rev. D 13 (1976) 974, Phys. Rev. D 19 (1979) 1277; L. Susskind, Phys. Rev. D 20 (1979) 2619; G. 't Hooft, Proceedings of the NATO Advanced Summer Institute, Cargese, 1979, edited by G. 't Hooft et. al (Plenum, New York, 1980) p. 135.

[21] G. Dvali and A. Pomarol, Phys. Rev. Lett. 77 (1996) 3738; P. Binetruy and E. Dudas, Phys. Lett. B 389 (1996) 503.

[22] Q. Shafi, Z. Tavartkiladze, Phys. Lett. B 473 (2000) 272.

[23] M. Dine, A. Kagan, S. Samuel, Phys. Lett. B 243 (1990) 250; L. Everett et al., Phys. Lett. B 477 (2000) 233; J. Feng, K. Matchev, hep-ph/0011356; J. Hisano, K. Kurosawa, Y. Nomura, hep-ph/0002286; See also references therein. 
[24] S. Dimopoulos, G. Giudice, Phys. Lett. B 357 (1995) 537; N. Arkani-Hamed, H. Murayama, hep-ph/9703259.

[25] J. Hisano, K. Kurosawa, Y. Nomura, Phys. Lett. B 445 (1999) 316.

[26] A. Nelson, D. Wright, Phys.Rev. D 56 (1997) 1598.

[27] M. Green and J. Schwarz, Phys. Lett. B 149 (1984) 117.

[28] M. Dine, N. Seiberg and E. Witten, Nucl. Phys. B 289 (1987) 584; J. Atick, L. Dixon and A. Sen, Nucl. Phys. B 292 (1987) 109; M. Dine, I. Ichinose and N. Seiberg, Nucl. Phys. B 293 (1987) 253.

[29] P. Ramond, hep-ph/9808489, and references therein; J. Sato and T. Yanagida, hepph/9809307; See also F. Vissani, hep-ph/9810435.

[30] D. Suematsu, Phys. Lett. B 392 (1997) 413; S. Davidson, S.F. King, hep-ph/9808296.

[31] P. Binetruy et al., Nucl. Phys. B 496 (1997) 3; V. Barger, T.J. Weiler and K. Whisnant, Phys. Lett. B 427 (1998) 97; Y. Grossman, Y. Nir and Y. Shadmi, hep-ph/9808355; Q. Shafi and Z. Tavartkiladze, Phys. Lett. B 448 (1999) 46, hep$\mathrm{ph} / 9811463$. In the context of realistic $S U(4)_{c} \times S U(2)_{L} \times S U(2)_{R}$ model with maximal $\nu_{\mu}-\nu_{\tau}$ mixing, it was also discussed in ref. [11].

[32] P. Binetruy , S. Lavignac, S. Petcov, Nucl. Phys. B 496 (1997) 3; Z. Berezhiani, Z. Tavartkiladze, Phys. Lett. B 396 (1997) 150; Phys. Lett. B 409 (1997) 220; Y. Grossman, Y. Nir, Y. Shadmi , JHEP 9810 (1998) 007; M. Fukugita, et al., hepph/9809554; M. Gomez et al., Phys. Rev. D 59 (1999) 116009; C. Froggat, M. Gibson, H. Nielsen, hep-ph/9811265; S. Kang, C. Kim, hep-ph/9811379; J. Feng, Y. Nir, hepph/9911370; J.M. Mira et al, Phys. Lett. B 492 (2000) 81; G. Altarelli et al., hepph/0007254; R. Mohapatra, hep-ph/0008232; M. Berger, K. Siyeon, hep-ph/0010245; I. Gogoladze, A. Perez-Lorenzana, hep-ph/0112034; see also references therein.

[33] J. Elwood, N. Irges, P. Ramond, hep-ph/9807228; F. Vissani, hep-ph/9810435; Z. Berezhiani, A. Rossi, hep-ph/9811447; R. Barbieri, L. Hall, G. Kane, G. Ross, hepph/9901228; S. Barr, I. Dorsner, hep-ph/0003058; J. Chkareuli, C. Froggatt, H. Nielsen, hep-ph/0109156; T. Kitabayashi, M. Yasue, hep-ph/0112287; F. Feruglio, A. Strumia, F. Vissani, hep-ph/0201291; H.J. He, D.A. Dicus, J.N. Ng, hep-ph/0203237; N.N. Singh, M. Patgiri, hep-ph/0204021; S.F. King, hep-ph/0204360; see also references therein.

[34] Q. Shafi, Z. Tavartkiladze, Phys. Lett. B 482 (2000) 145, hep-ph/0101350. 
[35] Q. Shafi, Z. Tavartkiladze, Phys. Lett. B 487 (2000) 145.

[36] Q. Shafi, Z. Tavartkiladze, Phys. Lett. B 448 (1999) 46.

[37] R. Barbieri at el., hep-ph/9807235; A.S. Joshipura, S.D. Rindani, hep-ph/9811252; R.N. Mohapatra, A. Perez-Lorenzana, C.A. de S. Pires, hep-ph/9911395; T. Kitabayashi, M. Yasue, hep-ph/0006014; K.S. Babu, R.N. Mohapatra, hepph/0201176. 